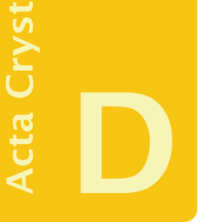

BIOLOGICAL

CRYSTALLOGRAPHY

Volume 71 (2015)

Supporting information for article:

Bond distances in polypeptide backbone depend on the local conformation

Roberto Improta, Luigi Vitagliano and Luciana Esposito 
Table S1 PDB codes for the set of very high-resolution protein structures used in the statistical survey of peptide bond geometrical parameters.

\begin{tabular}{ll}
\hline PDB code\# & Resolution (̊) \\
\hline 1EJG, 1UCS, 2VB1, 1US0, 2DSX, 1YK4, & $0.5-0.8$ \\
3A38, 1R6J, 1HJE, 2B97, 2OV0, 2WFI, & \\
3AL1, 4I8H, 1GCI, 1X6Z, 3W5H, 2PVE, & \\
1PQ7, 1W0N, 2IXT, 3UI4 & \\
\hline
\end{tabular}

1NWZ, 2H5C, 2VXN, 2JFR, 2O9S, 2PWA, $\quad 0.8-0.9$

1P9G, 2HS1, 2O7A, 2YKZ, 4EIC, 1M40,

1MC2, 1X8Q, 2F01, 2FMA, 2OL9, 3O4P,

3QPA, 4AYO, 1G6X, 1MUW, 2DDX, 3NJW,

3ZSJ, 1DY5, 1V6P, 3Q8J, 4HS1, 1GWE, 2P74,

3KLR, 3ZOJ, 4F1V, 1ETL, 1I1W, 3IP0, 1BYZ,

1ET1, 1G66, 1IX9, 1N9B, 1OEW, 1VYR, 2BW4,

2WUR, 2XU3, 3G21, 3KS3, 3VOR, 3ZR8, 4EA9

\# When multiple chains are present, chain A is selected. 
Table S2 Experimental distributions of peptide bond lengths for residues grouped by $\psi$ values. Ttest results for pairwise comparisons among means from adjacent bins are also reported (see main text).

\section{C-N statistics}

\begin{tabular}{|c|c|c|c|c|c|}
\hline C-N distance & $\begin{array}{l}40^{\circ} \text { bin centred } \\
\text { around } \psi=-\mathbf{6 0} 0^{\circ}\end{array}$ & $\begin{array}{c}40^{\circ} \text { bin centred } \\
\text { around } \psi=0^{\circ}\end{array}$ & $\begin{array}{l}40^{\circ} \text { bin centred } \\
\text { around } \psi=60^{\circ}\end{array}$ & $\begin{array}{l}40^{\circ} \text { bin centred } \\
\text { around } \psi=120^{\circ}\end{array}$ & $\begin{array}{l}40^{\circ} \text { bin centred } \\
\text { around } \psi=180^{\circ}\end{array}$ \\
\hline Mean & 1.3360 & 1.3319 & 1.3347 & 1.3315 & 1.3335 \\
\hline Variance $\left(\sigma^{2}\right)$ & 0.000100 & 0.000138 & 0.000126 & 0.000139 & 0.000147 \\
\hline Stand. Dev. $(\sigma)$ & 0.0100 & 0.0118 & 0.0112 & 0.0118 & 0.0121 \\
\hline Stand. Err. $(\sigma / \sqrt{ } N)$ & 0.00026 & 0.00044 & 0.00087 & 0.00028 & 0.00051 \\
\hline Sample size $(\mathrm{N})$ & 1468 & 703 & 168 & 1732 & 565 \\
\hline \multicolumn{6}{|l|}{ T-test results } \\
\hline C-N distance & $\psi=-60^{\circ} / \psi=0^{\circ}$ & $\psi=60^{\circ} / \psi=0^{\circ}$ & $\psi=60^{\circ} / \psi=120^{\circ}$ & $\psi=180^{\circ} / \psi=120^{\circ}$ & \\
\hline P-value $(\alpha=0.05)$ & $5.4 * 10^{-16}$ & 0.0026 & $5.2 * 10^{-4}$ & $4.3 * 10^{-4}$ & \\
\hline Diff. of means \# & yes & yes & yes & yes & \\
\hline
\end{tabular}

\section{C-O statistics}

\begin{tabular}{|c|c|c|c|c|c|}
\hline C-O distance & $\begin{array}{l}40^{\circ} \text { bin centred } \\
\text { around } \psi=-\mathbf{6 0} 0^{\circ}\end{array}$ & $\begin{array}{c}40^{\circ} \text { bin centred } \\
\text { around } \psi=0^{\circ}\end{array}$ & $\begin{array}{l}40^{\circ} \text { bin centred } \\
\text { around } \psi=60^{\circ}\end{array}$ & $\begin{array}{l}40^{\circ} \text { bin centred } \\
\text { around } \psi=120^{\circ}\end{array}$ & $\begin{array}{l}40^{\circ} \text { bin centred } \\
\text { around } \psi=180^{\circ}\end{array}$ \\
\hline Mean & 1.2353 & 1.2347 & 1.2339 & 1.2351 & 1.2324 \\
\hline Variance $\left(\sigma^{2}\right)$ & 0.0000901 & 0.000128 & 0.000098 & 0.000126 & 0.000119 \\
\hline Stand. Dev. $(\sigma)$ & 0.00949 & 0.0113 & 0.0099 & 0.0113 & 0.0109 \\
\hline Stand. Err. $(\sigma / \sqrt{ } N)$ & 0.00025 & 0.00043 & 0.00076 & 0.00027 & 0.00046 \\
\hline Sample size $(\mathrm{N})$ & 1468 & 703 & 168 & 1732 & 565 \\
\hline
\end{tabular}

\section{T-test results}

\section{C-O distance}

P-value $(\alpha=0.05)$

$\psi=0^{\circ} / \psi=-60^{\circ}$
0.105

Diff. of means \# $\quad$ no $\quad$ no $\quad$ no $\quad$ yes

\section{$\psi=120^{\circ} / \psi=60^{\circ}$ 0.0753}

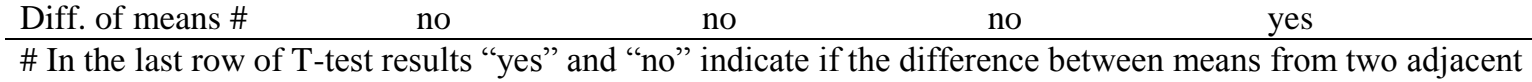

\section{$\psi=120^{\circ} / \psi=180^{\circ}$}

$4.0 * 10^{-7}$

\begin{tabular}{llcc} 
Diff. of means \# & no & no & no \\
\hline \# In the last row of T-test results "yes" and "no" indicate if the difference between means from two adjacent
\end{tabular} bins is statistically significant with the first mean (on the left) greater than the second (on the right) or not, respectively. 
Table S3 Experimental distributions of bond lengths at $\mathrm{C}^{\alpha}$ atom for residues grouped by $\psi$ values.

T-test results for pairwise comparisons among means from adjacent bins are also reported (see main text).

\begin{tabular}{|c|c|c|}
\hline $\mathrm{C}^{\alpha}-\mathrm{C}^{\beta}$ distance & $\begin{array}{l}40^{\circ} \text { bin centred } \\
\text { around } \boldsymbol{\psi}=\mathbf{- 3 0 ^ { \circ }}\end{array}$ & $\begin{array}{l}40^{\circ} \text { bin centred } \\
\text { around } \psi=60^{\circ}\end{array}$ \\
\hline Mean & 1.5312 & 1.5259 \\
\hline Variance & 0.000172 & 0.000146 \\
\hline Stand. Dev. $(\sigma)$ & 0.0131 & 0.0121 \\
\hline Stand. Err. $(\sigma / \sqrt{ } N)$ & 0.00026 & 0.00093 \\
\hline Sample size $(\mathrm{N})$ & 2609 & 168 \\
\hline \multicolumn{3}{|l|}{ T-test results } \\
\hline $\mathrm{C}^{\alpha}-\mathrm{C}^{\beta}$ distance & $\psi=-30^{\circ} / \psi=60^{\circ}$ & $\psi=150^{\circ} / \psi=60^{\circ}$ \\
\hline P-value $(\alpha=0.05)$ & $1.3 * 10^{-7}$ & $5.4 * 10^{-14}$ \\
\hline Diff. of means \# & yes & yes \\
\hline \multicolumn{3}{|l|}{$N-C^{\alpha}$ statistics } \\
\hline $\mathrm{N}-\mathrm{C}^{\alpha}$ distance & $\begin{array}{l}40^{\circ} \text { bin centred } \\
\text { around } \psi=0^{\circ}\end{array}$ & $\begin{array}{l}40^{\circ} \text { bin centred } \\
\text { around } \psi=90^{\circ}\end{array}$ \\
\hline Mean & 1.4559 & 1.4612 \\
\hline Variance & 0.000117 & 0.000131 \\
\hline Stand. Dev. $(\sigma)$ & 0.0108 & 0.0114 \\
\hline Stand. Err. $(\sigma / \sqrt{ } N)$ & 0.00041 & 0.00068 \\
\hline Sample size $(\mathrm{N})$ & 703 & 281 \\
\hline
\end{tabular}

\section{T-test results}

\begin{tabular}{lcc}
\hline $\mathrm{N}-\mathrm{C}^{\alpha}$ distance & $\boldsymbol{\psi}=\mathbf{9 0} / \boldsymbol{\psi}=\mathbf{0}^{\circ}$ & $\boldsymbol{\psi}=\mathbf{9 0 ^ { \circ }} / \boldsymbol{\psi}=180^{\circ}$ \\
\hline P-value $(\alpha=0.05)$ & $6.8 * 10^{-12}$ & $9.4^{*} 10^{-24}$ \\
Diff. of means \# & yes & yes \\
\hline
\end{tabular}

\section{$C^{\alpha}-C$ statistics}

\begin{tabular}{|c|c|c|}
\hline $\mathrm{C}^{\alpha}-\mathrm{C}$ distance & $\begin{array}{l}40^{\circ} \text { bin centred } \\
\text { around } \psi=-30^{\circ}\end{array}$ & $\begin{array}{l}40^{\circ} \text { bin centred } \\
\text { around } \psi=60^{\circ}\end{array}$ \\
\hline Mean & 1.5254 & 1.5306 \\
\hline Variance & 0.000130 & 0.000170 \\
\hline Stand. Dev. $(\sigma)$ & 0.0114 & 0.0131 \\
\hline Stand. Err. $(\sigma / \sqrt{ } N)$ & 0.00022 & 0.0010 \\
\hline Sample size $(\mathrm{N})$ & 2609 & 168 \\
\hline \multicolumn{3}{|l|}{ T-test results } \\
\hline $\mathrm{C}^{\alpha}-\mathrm{C}$ distance & $\psi=60^{\circ} / \psi=-30^{\circ}$ & $\psi=60^{\circ} / \psi=150^{\circ}$ \\
\hline P-value $(\alpha=0.05)$ & $7.5 * 10^{-7}$ & $4.4 * 10^{-10}$ \\
\hline Diff. of means \# & yes & yes \\
\hline
\end{tabular}

\# In the last row of T-test results "yes" and "no" indicate if the difference between means from two adjacent bins is statistically significant with the first mean (on the left) greater than the second (on the right) or not, respectively. 


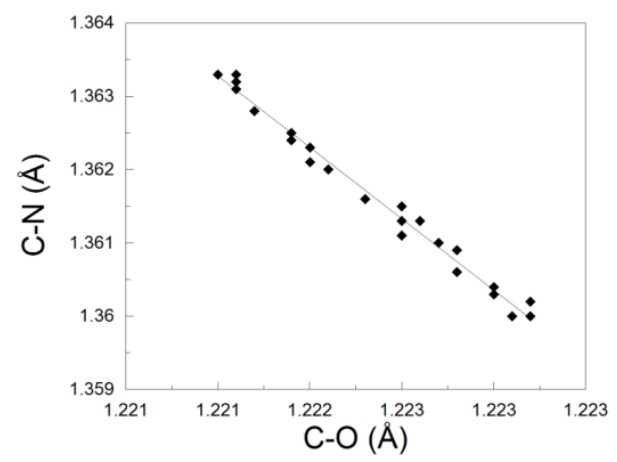

Figure S1 Pep model in vacuo: Correlation of C-O/C-N peptide bond distances. The regression line is also shown (correlation coefficient $\mathrm{R}=-0.99 ; \mathrm{y}_{\mathrm{C}-\mathrm{N}}=3.37-1.94 \mathrm{x}_{\mathrm{C}-\mathrm{O}}$ ).

(a)

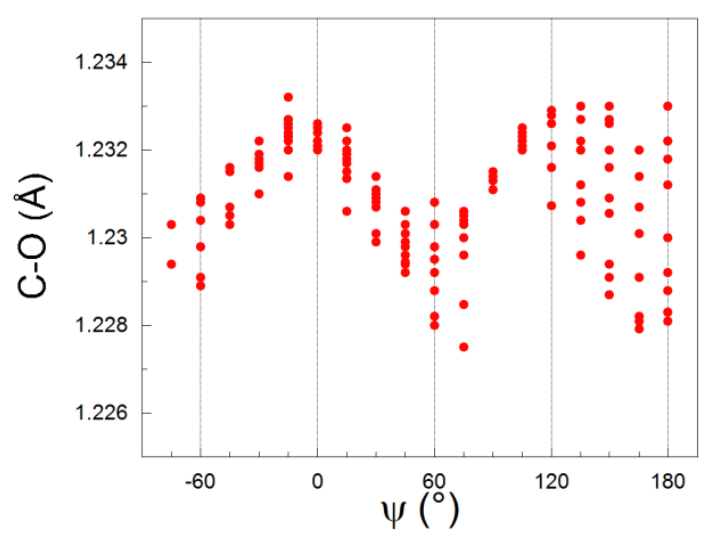

(c)

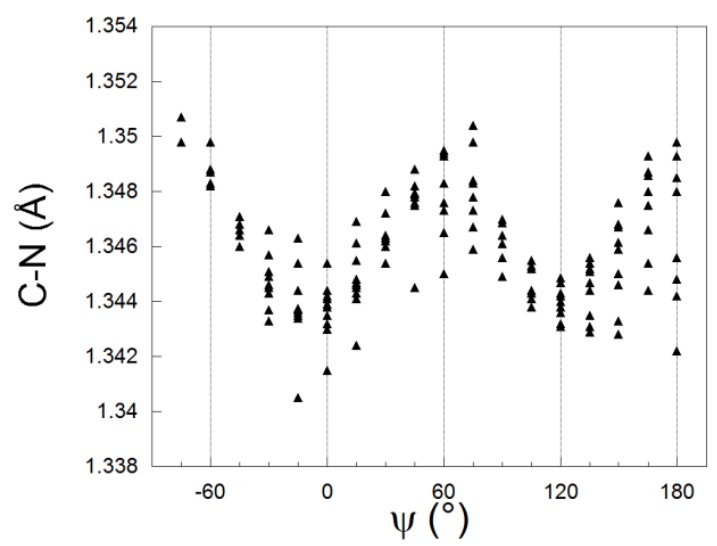

(b)

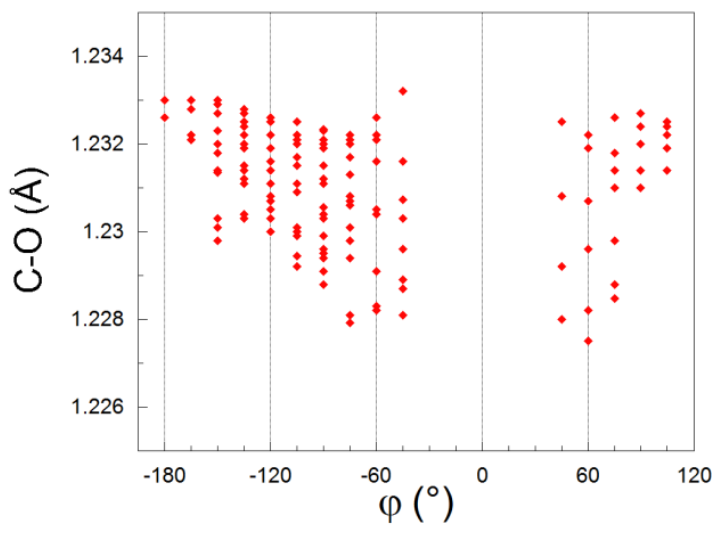

(d)

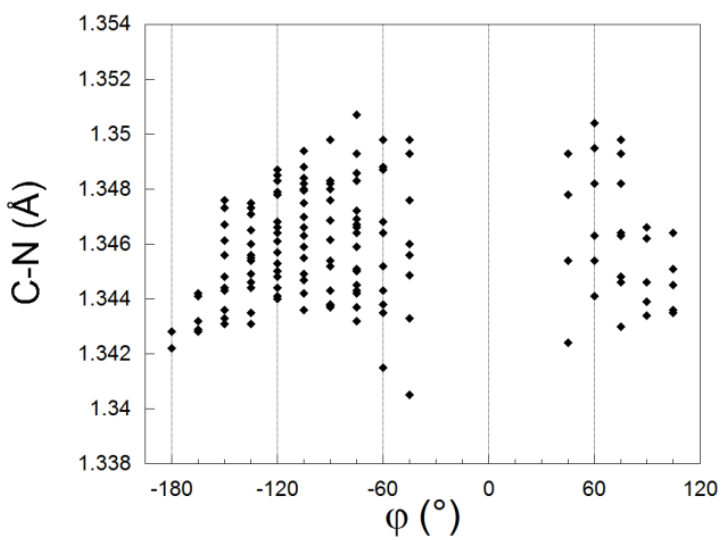

Figure S2 Ala1 model: Variability of $\mathrm{CO} / \mathrm{CN}$ bond distances with the $\psi$ and the $\varphi$ angle. (a) C-O vs. $\psi$. (b) C-O vs. $\varphi$. (c) C-N vs. $\psi$. (d) C-N vs. $\varphi$. 


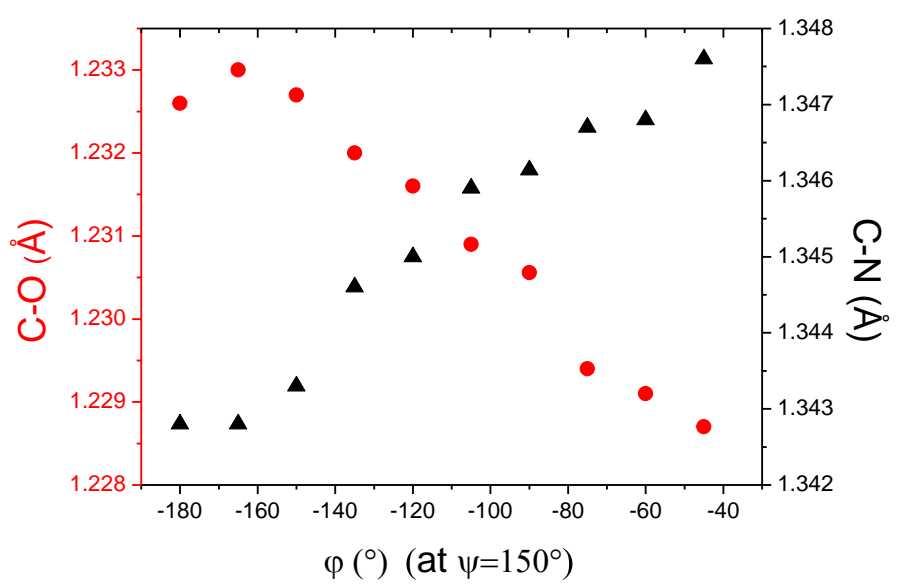

Figure S3 Ala1 model: Distance dependence on $\varphi$ in the extended conformation region (fixed $\psi=150^{\circ}$ ).

(a)

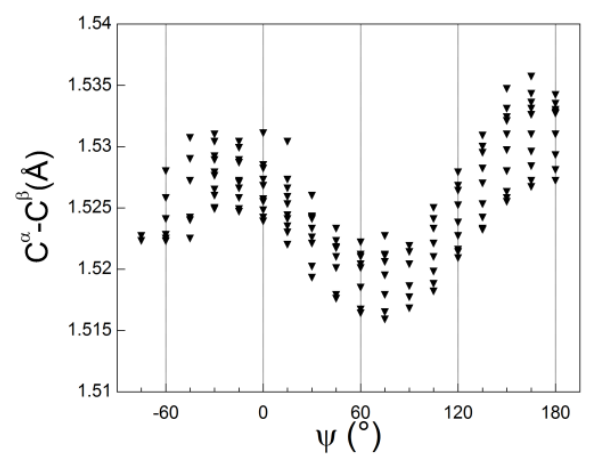

(c)

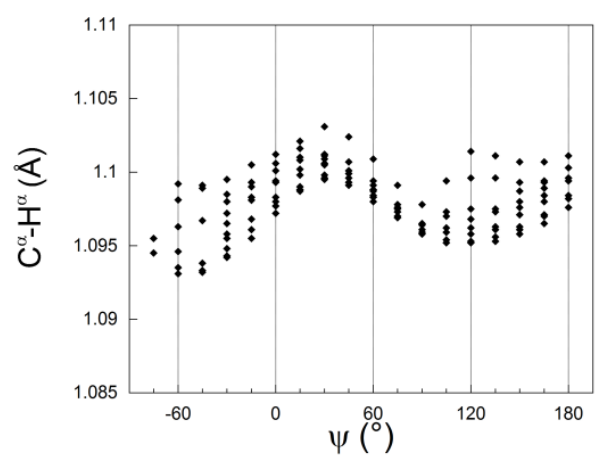

(b)

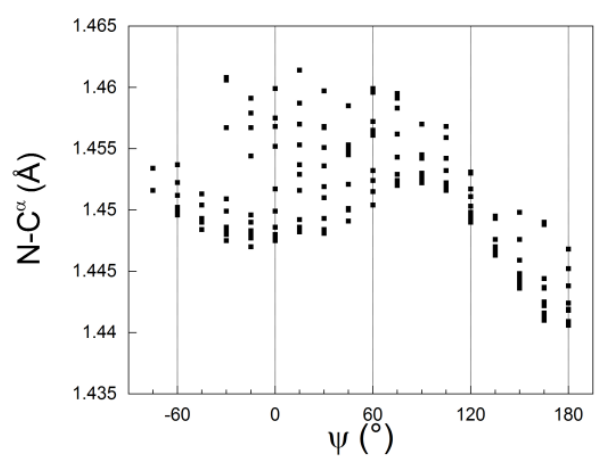

(d)

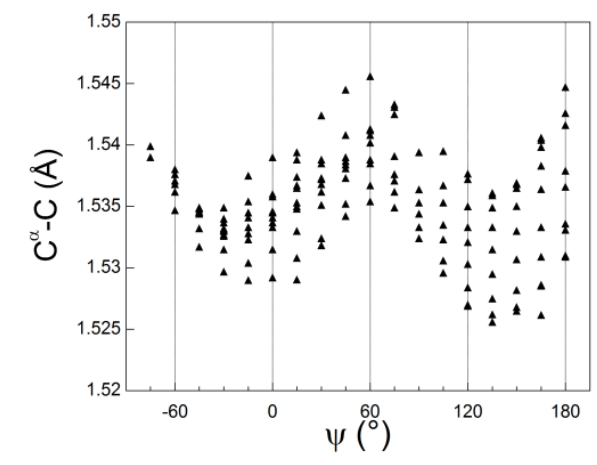

Figure S4 Ala1 model: Dependence of bond distances at $C^{\alpha}$ atom on the $\psi$ angle. $(a) C^{\alpha}-C^{\beta}$. (b) N$\mathrm{C}^{\alpha} .\left(\right.$ c) $\mathrm{C}^{\alpha}-\mathrm{H}^{\alpha}$. (d) $\mathrm{C}^{\alpha}-\mathrm{C}$. 
<smiles>[13CH3][13CH]=O</smiles>

$\psi=0^{\circ}$<smiles>N#CC(=O)S</smiles>

$\psi=-120^{\circ}$

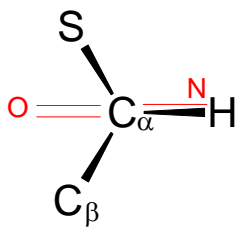

$\psi=120^{\circ}$<smiles>C[C@@H]([S-])C#N</smiles>

$\psi=180^{\circ}$<smiles>C[C@H](O)S</smiles>

$\psi=-60^{\circ}$<smiles>N#CC(=O)S</smiles>

$\psi=60^{\circ}$<smiles>N[C@H](S)C=O</smiles>

$\psi=-150^{\circ}$

$\psi=30^{\circ}$<smiles>N[C@H](S)CS</smiles><smiles>[14CH3][14CH2][14CH2][14CH2][14CH2][14CH2]N</smiles>

$\psi=150^{\circ}$<smiles>[13CH3]C([13CH3])S</smiles>

$\psi=90^{\circ}$<smiles>N[C@H](S)C=O</smiles>

$\psi=-90^{\circ}$

Figure S5 Schematic drawings of conformers characterized by different $\psi$ values. The projections are drawn by looking along the $\mathrm{C}^{\alpha}-\mathrm{C}$ bond. The $\mathrm{S}$ substituent stands for the $\mathrm{CH}_{3}-\mathrm{CO}-\mathrm{NH}$ - group in Ala1 model. Going from left to right: conformers with one $\mathrm{C}^{\alpha}$ bond (i) eclipsed with the C-N bond, (ii) eclipsed with $\mathrm{C}-\mathrm{O}$ bond, (iii, iv) perpendicular to the $\mathrm{CO}-\mathrm{NH}$ plane. The figure is adapted from Fig S2 of Improta et al. 2011. 
(a)

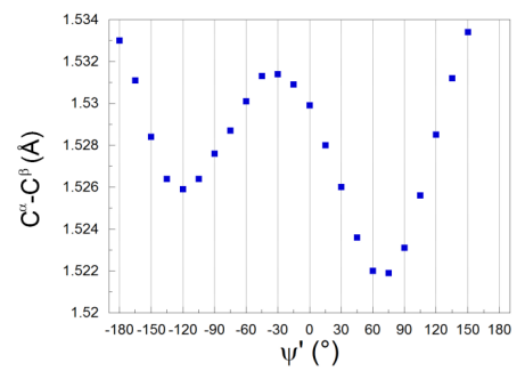

(b)

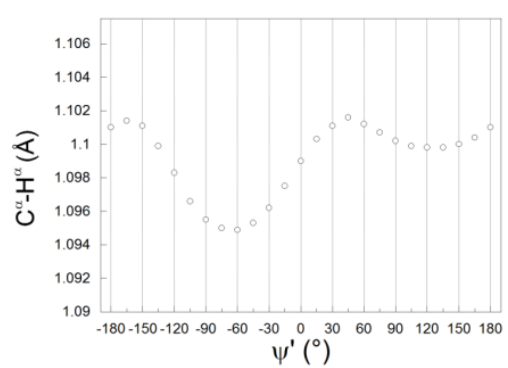

(c)

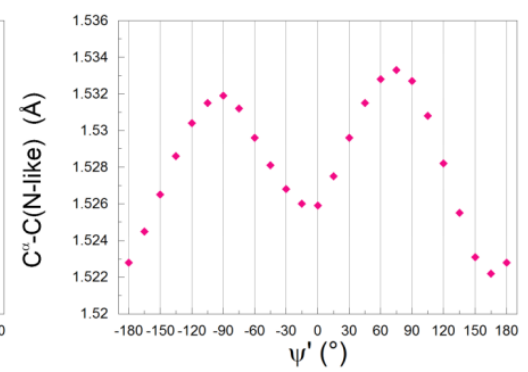

Figure S6 Pep model: Dependence of bond distances at $C^{\alpha}$ atom on the $\psi^{\prime}$ angle. $(a) C^{\alpha}-C^{\beta}$. (b) $C^{\alpha}$ $\mathrm{H}^{\alpha}$. (c) $\mathrm{C}^{\alpha}-\mathrm{CH}_{3}(\mathrm{~N}-$-like $)$.

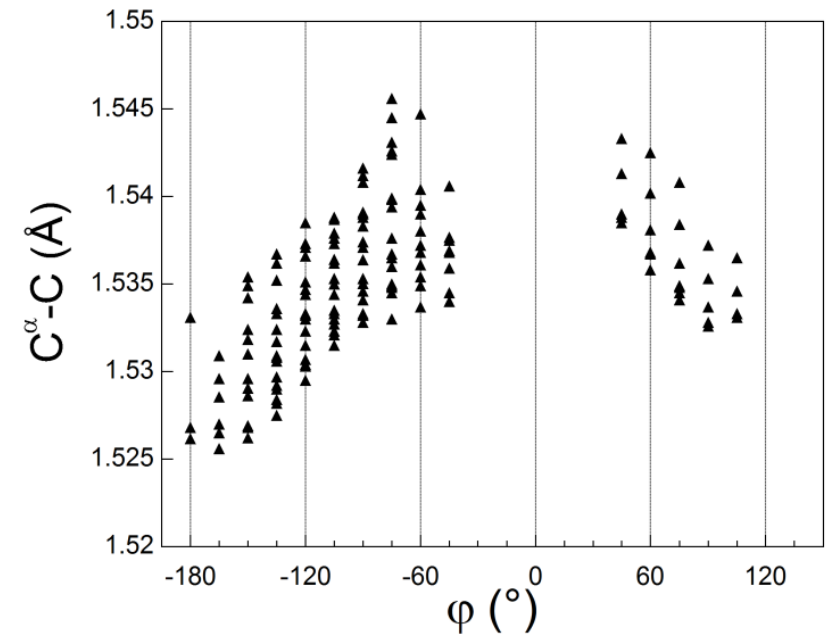

Figure S7 Ala1 model: Dependence of $\mathrm{C}^{\alpha}-\mathrm{C}$ bond distance on the $\varphi$ angle. 
(a)

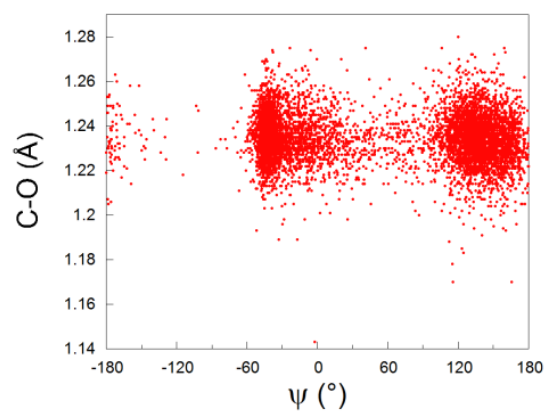

(c)

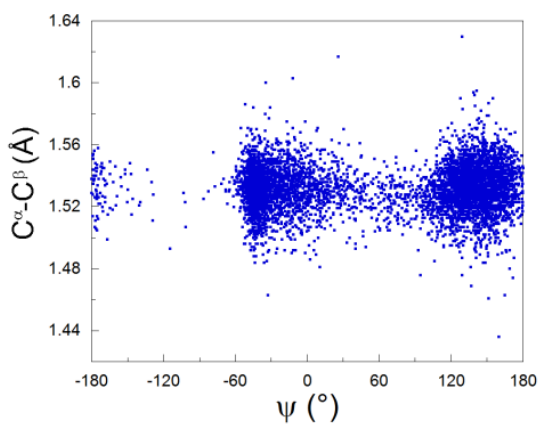

(b)

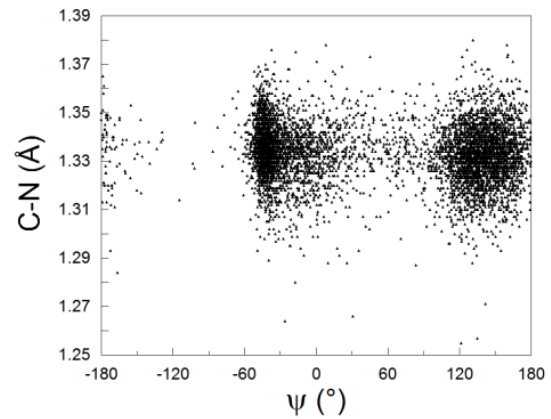

(d)

(e)
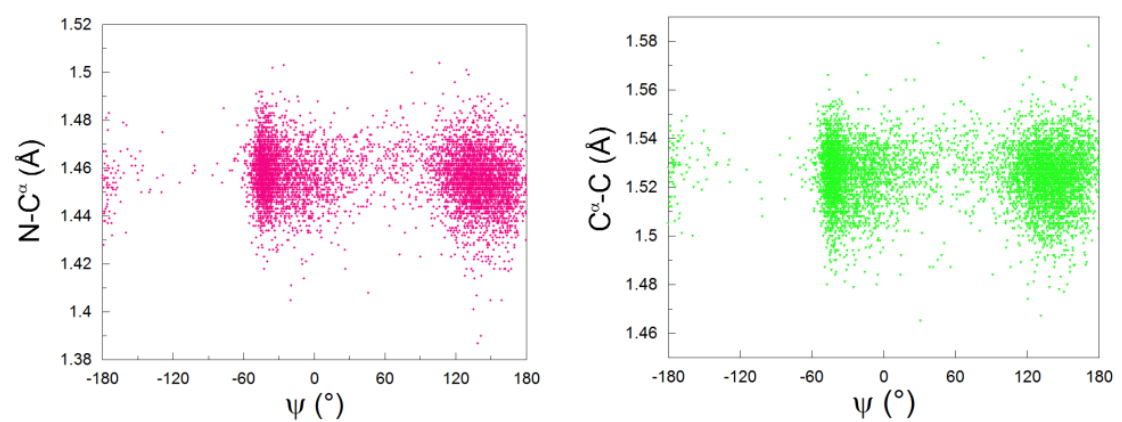

Figure S8 PDB ultrahigh resolution protein structure survey. Dependence of bond lengths on $\psi$ dihedral angle. (a) C-O vs. $\psi$. (b) C-N vs. $\psi$. (c) $\mathrm{C}^{\alpha}-\mathrm{C}^{\beta} v s . \psi .(d) \mathrm{N}-\mathrm{C}^{\alpha} v s . \psi \cdot(e) \mathrm{C}^{\alpha}-\mathrm{C}$ 
(a)

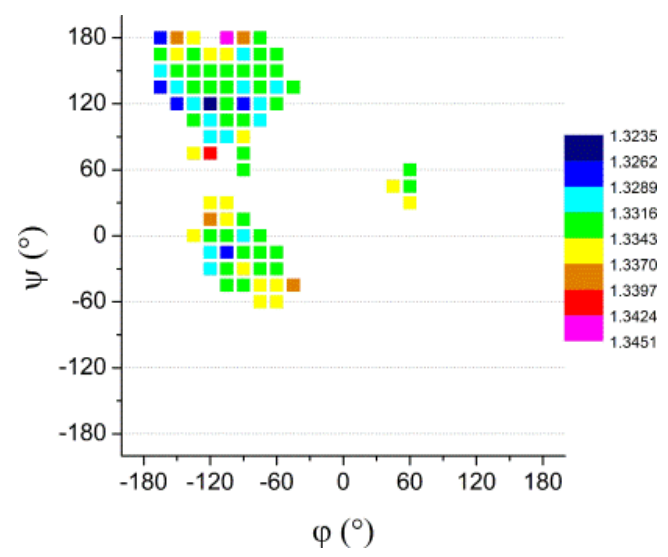

(b)

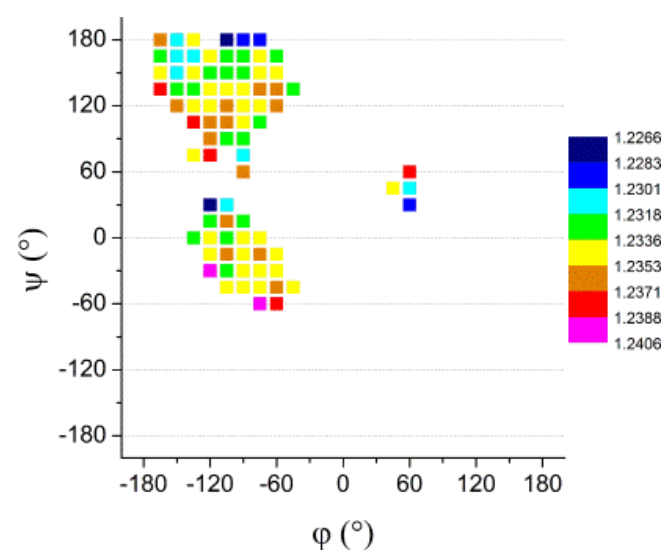

(c)

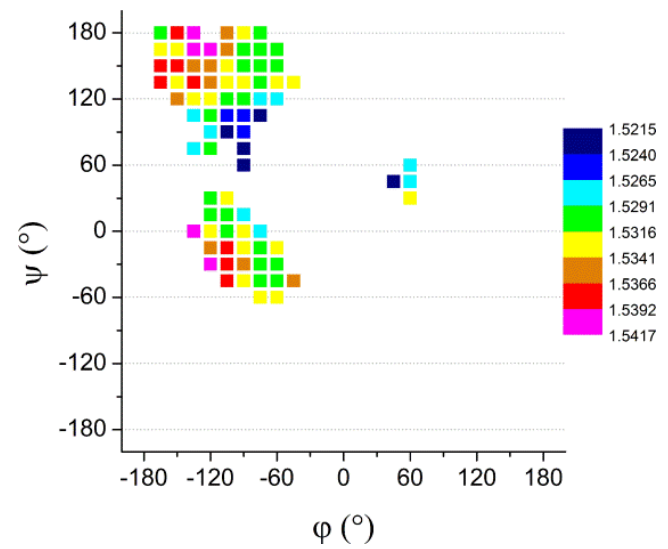

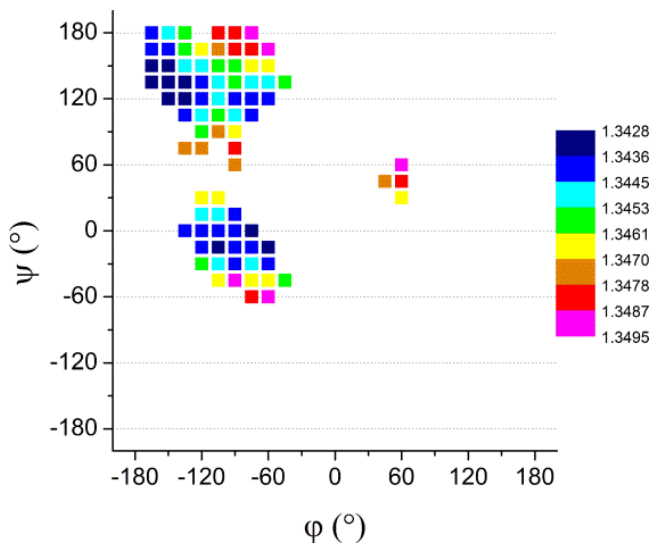
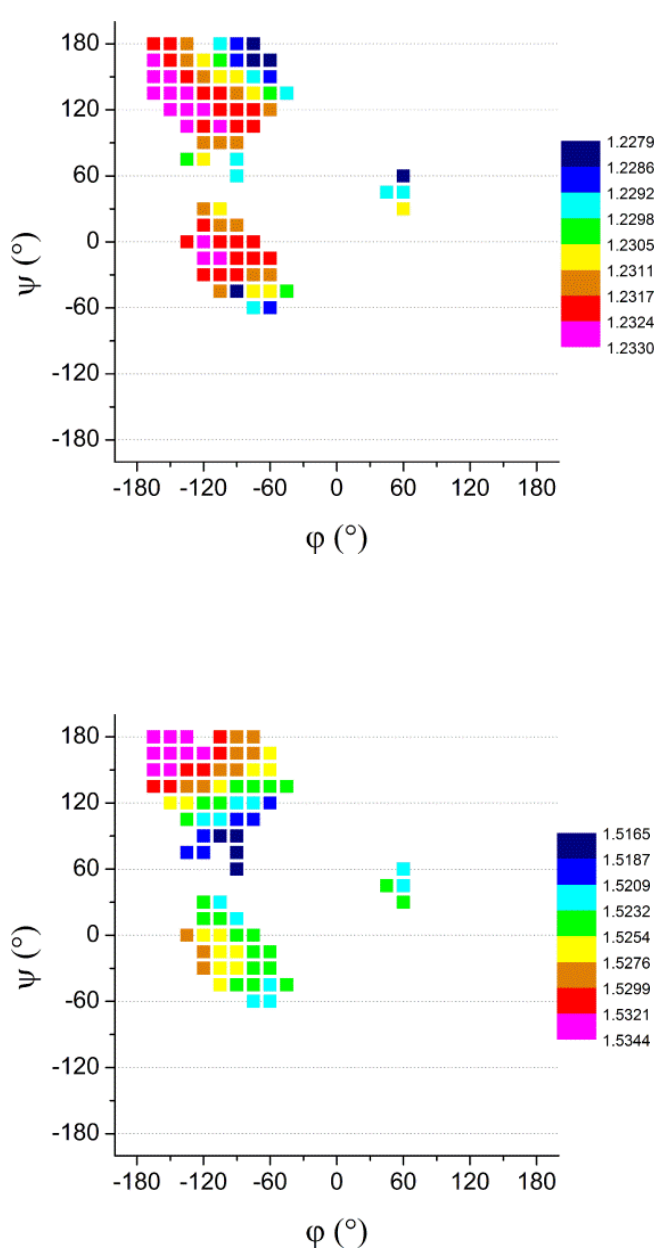
(d)
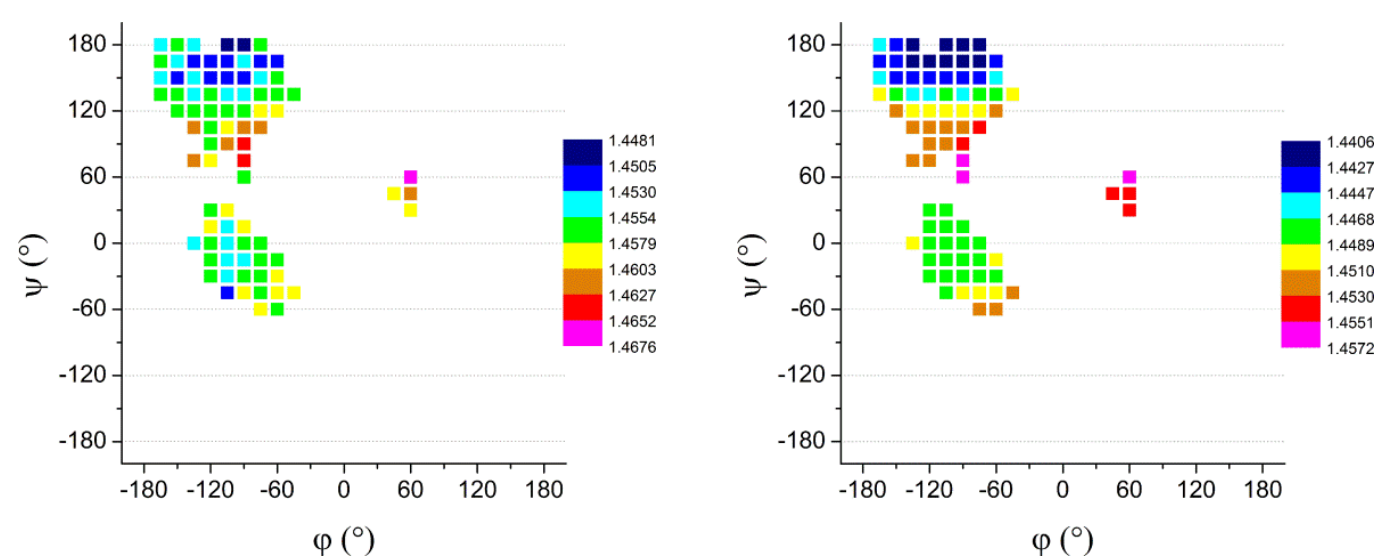

(e)
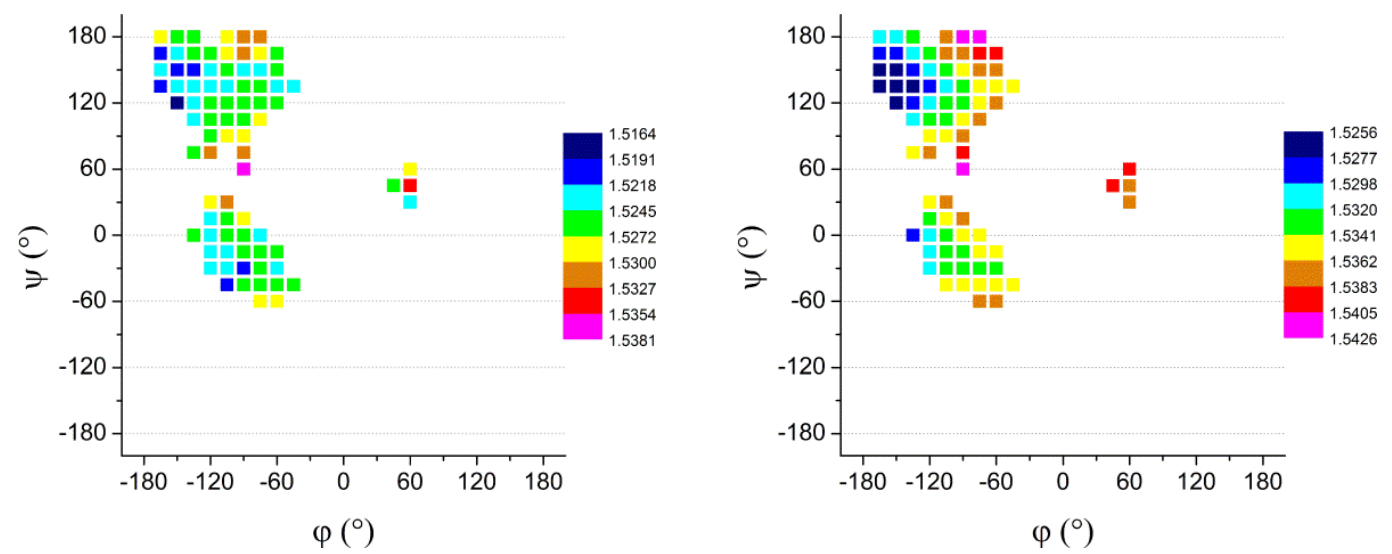

Figure S9 Comparison of conformational dependences of distance lengths. Results from experimental structures (left) and theoretical calculations (right). The experimental values are calculated by averaging the distances (all residue types except Gly) in $(\varphi, \psi)$ bins of $\left(15^{\circ} \times 15^{\circ}\right)$ size, centred on fixed values used for calculations. Only bins containing more than 10 measures are considered. (a) C-N, (b) C-O, (c) $\mathrm{C}^{\alpha}-\mathrm{C}^{\beta},(d) \mathrm{N}-\mathrm{C}^{\alpha},(e) \mathrm{C}^{\alpha}-\mathrm{C}$. 
a)

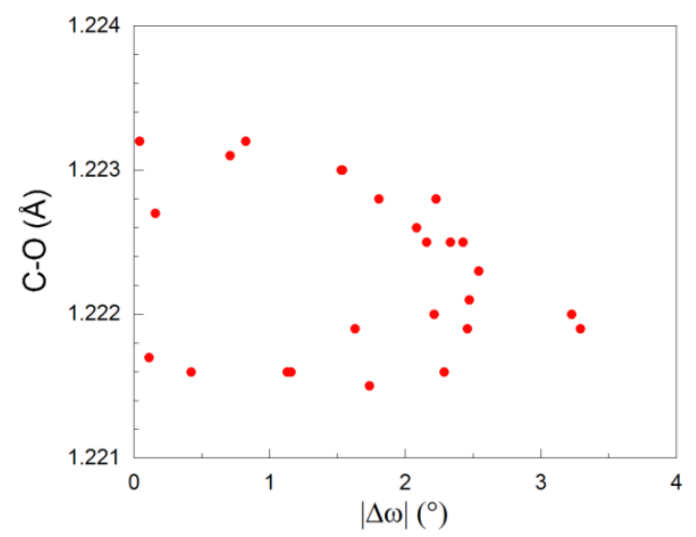

(b)

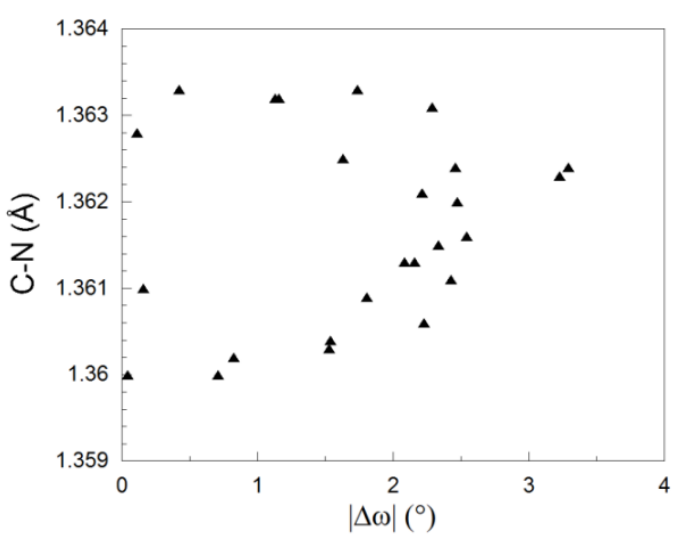

Figure S10 Pep model in vacuo: $\mathrm{CO} / \mathrm{CN}$ distances versus $|\Delta \omega|$. (a) CO versus $|\Delta \omega|$. (b) $\mathrm{CN}$ versus $|\Delta \omega|$. It is worth noting that $\Delta \omega$ is defined as follows: $\Delta \omega=\omega-180^{\circ}\left(\bmod 360^{\circ}\right)$. 